used to capture two components of self-regulation - task attentiveness and emotional regulation. Children who had lived in poverty at 9 months were almost twice as likely to smoke at age 14. Twelve per cent of the difference in smoking between poverty groups was attributable to self-regulation, with none explained by verbal ability. The remaining difference was largely due to socio-demographic factors. Interventions to improve self-regulation targeted at children from families experiencing socioeconomic disadvantage at the end of primary school may hold promise to reduce inequalities in smoking. However, the detrimental impact of early life poverty on smoking in adolescence is chiefly driven by socio-demographic factors rather than later, individual characteristics such as self-regulation.

\section{P26 EXPLORING THE EXPERIENCE OF ADOLESCENTS WITH DOWN SYNDROME AND THEIR PARENTS}

F McGrane*, EF Roche. 'Discipline of Paediatrics, Trinity College, Dublin, Dublin, Ireland; ${ }^{2}$ Department of Paediatrics, Children's Health Ireland, Tallaght, Dublin, Ireland

\subsection{6/bmjpo-2019-RCPCH-SAHM.31}

Aims Research regarding adolescents' with Down syndrome (DS) is limited. The majority of previous studies have been undertaken in either the UK or USA but none in Ireland. This study sought to address this gap by exploring the experience of growing up in Ireland for adolescents with Down syndrome and their aspirations for the future.

Methods The study was conducted using two questionnaires designed for administration to adolescents with Down syndrome and self-completion by parents. Areas explored in detail included health, service provision, education, living circumstances and future aspirations.

Results Forty seven adolescents with DS participated, aged 12-18 years (23 female) and 49 parents. The frequency of health comorbidities were assessed, $87 \%$ felt they had good health with eyes, hearing and heart. Further detail was obtained in key specific health areas. Daily medication was taken by $44 \%$. A large proportion of parents $(45 \%)$ and adolescents (98\%) were unaware of the DS medical management guidelines. Parents were asked if their adolescent received satisfactory services across the domains. Parental reported satisfaction with care received in vision (92\%), hearing (90\%), medical care (86\%) dietetics $(55 \%)$ and speech and language therapy (39\%). Dissatisfaction related to access rather than care received. There was limited use of respite services (30\%). There was a negative perception of respite amongst some parents (17\%) although those who availed of respite had largely positive experiences (92\%). Transition of care from paediatric to adult services is an area of concern for the majority of parents $(63 \%(n=31))$ but not for adolescents. Most adolescents $(79 \%)$ were unaware of transition to adult services and were unconcerned by it. Parents felt transition could be improved by additional information (61\%), increased preparation $(51 \%)$ and planning and discussion with adolescents $(63 \%)$.

Conclusion The study showed overall satisfaction in relation to health and service provision but highlighted concerns regarding access to critical time sensitive services. Of particular concern there was poor awareness of the Medical management guidelines.

\section{P27 \\ WHEN CHRONIC FATIGUE SYNDROME LEADS TO MUTISM}

${ }^{1,2} \mathrm{~S}$ Moeda*, 1,3 L Gamper, ' A Gregorowski, 'T Segal. 'Children and Young People's Specialist Adolescent Services, University College Hospital, London, UK; ${ }^{2}$ Department of Paediatrics, Hospital Beatriz Ângelo, Loures, Portugal; ${ }^{3}$ Department of Paediatrics, University Children's Hospital, Zurich, Switzerland

\subsection{6/bmjpo-2019-RCPCH-SAHM.32}

Aims Chronic Fatigue Syndrome (CFS) impacts negatively the quality of life, school attendance and social and family functioning. Cognitive dysfunction represents one of the most common and disabling symptoms. We report a severe case of CFS with extreme cognition loss.

Methods Case-report.

Results A previously healthy and athletic male adolescent developed weakness and fatigue following a viral illness at 10 years old. Mobility progressively deteriorated over two years, requiring wheelchair and admission. Investigations, including brain and spinal MRI, were unremarkable and CFS diagnosed. $\mathrm{He}$ started intensive physiotherapy but, while his physical activity improved, an abrupt extreme cognitive loss took place. He reported 'brain fog' and formal testing showed incredibly slow cognitive and sensory processing (e.g. score of 4/10 in processing incoming language). After little improvement, he restarted deteriorating and stopped attending school.

Aged 16, he was referred to our specialist CFS team, lying mainly in bed and unable to feed himself. He had diminished ability to speak and slow comprehensive processing. $\mathrm{He}$ struggled with significant anxiety, low mood, poor sleep and socially isolation. Further investigation, with EEG and neurological reassessment, was normal. He initiated a regular multidisciplinary rehabilitation program, including patient and family support, pacing, graded exercise, sleep hygiene and pain relief. He indicated that he chose to use his energy on physical improvement at the expense of communication, hence, while his mobility slowly improved over one year, his cognitive ability notably deteriorated. He became gradually more non-verbal and uncommunicative, initially using signboards, but then refusing. Once his physical activity had markedly improved from being bedbound to walking, he restarted communicating by smiling and using thumbs up/down, slow speech with lags between words, then rapidly increasing to full sentences. After two years of therapy his improvement has been remarkable. Currently he walks, sleeps regularly, speaks fluently and engages with family and friends. He has home- tuition and has future goals.

Conclusion Establishing the accurate diagnosis and early symptom management are crucial for rehabilitation in CFS. Cognitive impairment may be significantly debilitating. Physical and cognitive recovery occurs at different stages. A multi-disciplinary patient-centred approach, working with families and local services, can be very effective.

\section{P28 IMPROVING TRANSITION FOR PATIENTS WITH DIABETES: A SERVICE IMPROVEMENT PROJECT}

F Fuller*, R Gadsby. Warwick Medical School, Warwick University, Warwick UK

\subsection{6/bmjpo-2019-RCPCH-SAHM.33}

Aims To examine what adolescents with Type 1 diabetes want from a transition service and to evaluate if the implementation of a Specialist Transition Nurse improves patient engagement 